\title{
Artificial Bee Colony Algorithm for Fresh Food Distribution without Quality Loss by Delivery Route Optimization
}

\author{
Sapna Katiyar, ${ }^{1}$ Rijwan Khan $\mathbb{D}^{1},{ }^{1}$ and Santosh Kumar $\mathbb{D}^{2}$ \\ ${ }^{1}$ ABES Institute of Technology, Ghaziabad, Uttar Pradesh, India \\ ${ }^{2}$ University of Dar Es Salaam, Dar Es Salaam, Tanzania \\ Correspondence should be addressed to Santosh Kumar; drsengar2002@gmail.com
}

Received 9 June 2021; Revised 15 July 2021; Accepted 4 October 2021; Published 15 October 2021

Academic Editor: Alessandro Di Cerbo

Copyright ( 2021 Sapna Katiyar et al. This is an open access article distributed under the Creative Commons Attribution License, which permits unrestricted use, distribution, and reproduction in any medium, provided the original work is properly cited.

This paper enlightens the use of artificial intelligence (AI) for distribution of fresh foods by searching more viable route to keep intact the food attributes. In recent years, very hard-hitting competition is for food industries because of the individuals living standards and their responsiveness for fresh food products demand within stipulated time period. Food industry deals with the extensive kind of activities such as food processing, food packaging and distribution, and instrumentation and control. To meet market demand, customer satisfaction, and maintaining its own brand and ranking on global scale, artificial intelligence can play a vibrant role in decision-making by providing analytical solutions with adjusting available resources. Therefore, by integrating innovative technologies for fresh food distribution, potential benefits have been increased, and simultaneously risk associated with the food quality is reduced. Time is a major factor upon which food quality depends; hence, time required to complete the task must be minimized, and it is achieved by reducing the distance travelled; so, path optimization is the key for the overall task. Swarm intelligence (SI) is a subfield of artificial intelligence and consists of many algorithms. SI is a branch of nature-inspired algorithm, having a capability of global search, and gives optimized solution for real-time problems adaptive in nature. An artificial bee colony (ABC) optimization and cuckoo search (CS) algorithm also come into the category of SI algorithm. Researchers have implemented ABC algorithm and CS algorithm to optimize the distribution route for fresh food delivery in time window along with considering other factors: fixed number of delivery vehicles and fixed cost and fuel by covering all service locations. Results show that this research provides an efficient approach, i.e., artificial bee colony algorithm for fresh food distribution in time window without penalty and food quality loss.

\section{Introduction}

Fresh farm foods are perishable and dependent on time; hence, quality of food and its protection are the vital factors. To intact the food quality and avoiding its contamination, time required for shipping fresh food products must be less, and shipping environment must be safe. Therefore, the requirement of rational mechanism of logistic delivery of fresh farm foods arises which is of major concern for almost all customers [1,2]. China developed cold chain logistics, and various developments have also been done depending upon requirements and concerns, but at some point of time, it has been identified that this was not sufficient. Because of the challenges faced during guaranteed distribution of fresh food, one solution has been identified which is the path planning of delivery of food products even without considering the cold chain distribution. Path planning of food products distribution can improve the guarantee of quality food, customer satisfaction, avoiding food contamination, reducing logistic cost etc., which is the significant proposal for this research paper.

Vehicle routing problem (VRP) deals with searching a best path from source to destination by serving a number of customers in a sequence where time window is the critical 
component. VRP was first proposed in year 1959 by Dantzig, and later on in the year 1987, then in 1989 and till now (year 2020), many others also studied and designed. In research, VRP is the most deliberated combinatorial optimization problems [3, 4]. Various variants of vehicle routing problem exist which have been developed one after another, and still researchers are exploring some new dimensions. In recent years, various heuristic methods, evolutionary algorithm, and hybrid optimization algorithms have been successfully applied for VRP [5] and have given quality solutions along with computational quickness. In research, some vehicle routing problems for supplying fresh agricultural foods and frozen foods have been applied with time window constraints [6, 7], but in some most of the cases, food contamination during transportation was not taken into consideration.

Optimization algorithms have replaced the traditional techniques to serve the purpose because of various appealing features to deploy in real-time problems for accomplishing various tasks as they are efficient, can serve customer in better way with lots of clarity, and follow all predefined rules and increased efficiency. They can be improved with time, or modifications can be done depending upon the nature of problem/application [8-10]. Optimization is very much essential in the business domain because of optimization, and only cost can be reduced with increased performance. Therefore, profit can be increased. Application of optimization algorithm in food industry has also proven and setup a benchmark for others by using best practices and techniques [11]. Motivation for the research is to accomplish distribution of fresh food without quality degradation, and it can be achieved if time required for distribution can be reduced.

In recent years, various novel optimization algorithms such as ant colony optimization (ACO) [12, 13], particle swarm optimization (PSO) [14], cuckoo search (CS) $[15,16]$, lion optimization algorithm (LOA) [17], grasshopper optimization algorithm [18], Levy flight algorithm [19], tree growth algorithm [20], grey wolf optimizer [21], bees pollen optimization algorithm [22], Harris Hawks optimization (HHO) [23], sine cosine algorithm [24], water wave optimization algorithm [25], whale optimization algorithm [26], moth search (MS) [27], elephant herding optimization (EHO) [28], slime mould algorithm (SMA) [28], kill heard [29], and butterfly optimization (BO) algorithm [30], and hybrid algorithms have drawn the attention of researchers for applying these algorithms in various unconstrained and constrained optimization problems. Upon studying all mentioned algorithms, it has been identified that they have been successfully applied for test suite optimization, path convergence-based optimization, and many other real-world engineering problems. One of the very interesting and challenging real-time problems is fresh food distribution because it has to meet various standards with available resources constraints and customer satisfaction is the most important requirement.

One of the novel heuristic algorithms is artificial bee colony (ABC) algorithm, inspired by bee's behavior which has been applied to get a solution of complex combinational optimization problems. Every optimization algorithm has certain advantages, but $\mathrm{ABC}$ algorithm is preferred by the researcher for adaptive nature of the problem over other algorithms because of less control parameters, fast convergence, robust nature, highly flexible, and simple approach within stipulated time duration. The quality of bee colony is to search nectar spontaneously and to do so sometimes they divide themselves into three groups: leaders, onlooker, and scouts. Among these three characters, information is communicated and colony collects the nectar rapidly and proficiently. ABC algorithm and some of its variants have been applied effectively in various problems such as assignment problems with neighbourhood mechanism, scheduling problem, integrated portioning, Schottky barrier diode, and image segmentation [31, 32]. Such fruitful applications of artificial bee colony optimization algorithm encouraged authors to implement in this paper. The objective of this paper is to apply vehicle routing problem with time windows (VRPTW) to maximize the probability of timely delivery of food product to customers by minimizing the number of vehicles required, distance travelled by each vehicle, cost involved in transportation, and other logistic requirements. Results achieved are compared with results extracted from the cuckoo search (CS) algorithm. CS algorithm has a slow rate of convergence but is compatible with the ABC algorithm in terms of other parameters and performance. Upon compassion, it has been observed that the proposed algorithm, i.e., artificial bee colony optimization algorithm looks like an effective and competent method for the defined problem.

Organization of the paper is as follows: food quality, food categorization, and preservation are presented in Section 2. Artificial bee colony (ABC) algorithm is defined in Section 3. Cuckoo search (CS) algorithm is described in Section 4. Vehicle routing problem (VRP) and its variants are discussed in Section 5. Problem description and implementation are presented in Section 6. Results are illustrated in Section 7. Lastly, conclusion and future scope are enumerated in Section 8.

\section{Food Quality, Food Categorization, and Preservation}

Food makes our body to work, to grow, and to repair itself whenever required. Basically, food is an organic substance and consumed for nutritional purpose, and it comes from animals and plants. Food is eaten by living things to become healthy, and it contains protein, moistness, carbohydrate, minerals, fat, and other organic ingredients $[2,6]$.

2.1. Food Quality. The objective of food business is to endure competitive in global market by maintaining food quality. The term food quality is a very important factor especially in the success of a food product. This term consists of addition of all properties and features of food items which must be acceptable to all buyers. The term "food quality" is frequently used by consumers, but its value varies from customer to customer even among various regions and cultures. 
Generally, quality is subjective in nature; therefore, some reference is required for specific criteria or attributes. In food industry, product specifications are defined in view of quality attributes and specifications.

The attributes contributing to food quality are as follows:

(i) Appearance (it includes shape, size, color, shine, and consistency)

(ii) Nutritional content

(iii) Flavour

(iv) Texture

(v) Decent and viable production

2.2. Food Categorization. Figure 1 shows the classification of food and details about each category, and its subcategory is also explained in this section.

2.2.1. Categorization of Food Based on Shelf Life. Food spoilage with respect to time is a natural procedure because during this stage, food may progressively lose its color, odor, consistency, and nutritional values. In case someone consumed spoiled food, it can lead to mild to severe sickness till death also. In the basis of the self-life, food items can be categorized as follows:

(i) Nonperishable foods such as flour, nuts, and dry beans

(ii) Semiperishable foods such as cheese, vegetables, and fruits

(iii) Perishable foods such as milk, eggs, and dairy products

2.2.2. Categorization of Food Based on Functions. As per the functioning of the human body, it is categorized is as follows:

(i) Protective foods

(ii) Energy-giving foods

(iii) Body-building foods

(iv) Regulatory foods

2.2.3. Categorization of Food Based on Nutritional Values. On the basis of nutritional value, food items categorized are as follows:
(i) Protein-rich food
(ii) Vitamin-rich food
(iii) Mineral-rich food
(iv) Carbohydrate-rich food
(v) Fat-rich food

2.2.4. Categorization of Food Based on Extent and Purpose of Processing. Food industries try to convert fresh foods into food products. Therefore, various food processing techniques are utilized. In this category, further categorization is as follows: (i) Marginally processed or unprocessed food

(ii) Processed cookery food

(iii) Ultraprocessed food

2.3. Food Preservation. It is always required to maintain food quality along with its nutritive values, color, consistency etc., for longer duration of time because food contamination is very dangerous. It can have serious apprehensions like sickness to increased level even till death also. Therefore, legal responsibilities lie with food managers or supervisors that they must ensure that food does not contain any type of contaminations which may impact customer health. In any such type of severe conditions, serious penalty may be bear by the food business owner which may impact business reputation also.

Food preservation is a procedure via which internal and external features of food is maintained to avoid food decay. The objective is to intact its nutritional values, consistency, color, and taste. Food preservation had been started long time ago in olden days. Figure 2 shows the classification of food preservation. Traditional old methods of preservation are drying in sun, cooling, chilling, and fermentation. With technological development, recent methods of food preservation also include addition of preservatives like some chemicals or natural preservatives.

\section{Artificial Bee Colony (ABC) Algorithm}

An artificial bee colony ( $A B C)$ algorithm is a stochastic algorithm based on popular swarm intelligence and was proposed by Karaboga in 2007 for solving various optimization problems. This algorithm mimics the actions of intelligent honey bees with an objective to search the source of food. In a colony, bees are divided into three groups depending upon their roles [32, 33]:

(i) Employed bees: also known as forager bees; they go to search for rich food sources and after returning back to squirrel, they exchange the information with observer bees with some probability.

(ii) Onlooker bees: also known as observer bees; these are a kind of unemployed bees which start searching while scouts initiate in its nest or in neighbourhood. Exploitation search is carried out.

(iii) Scout bees: they interpret from some forager bees which abandon their sources and start searching new. Exploration search is carried out by them.

In artificial bee colony optimization algorithm, location of food source is treated as the possible solution of the given problem and amount of fluid (nectar) of food sources as fitness, i.e., quality of related solution. One employed bee corresponds to one food source; therefore, the total number of employed bees is the same as the number of solutions, i.e., food sources.

$\mathrm{ABC}$ algorithm executes four different selection processes:

(1) Global selection process: it is used by onlookers 


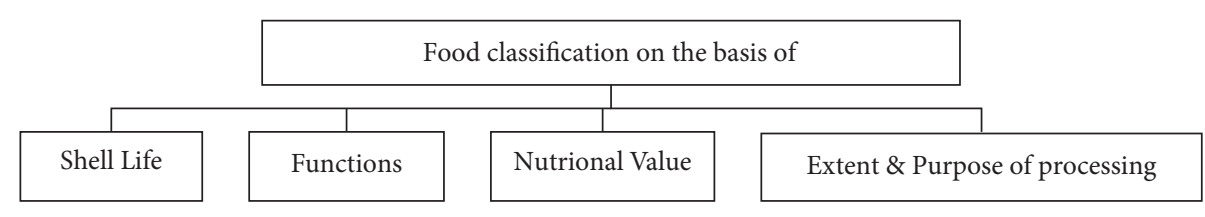

FIgURE 1: Classification of food preservation.

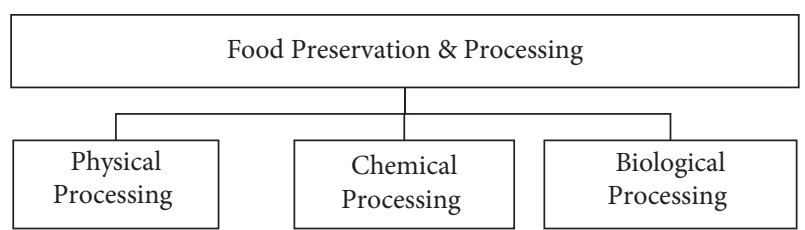

Figure 2: Classification of food preservation.

(2) Local selection process: it is carried out in a region by employed and onlooker bees

(3) Greedy selection process: it is used by all bees

(4) Random selection process: it is used by scouts

3.1. Organization of Artificial Bee Colony Algorithm. All the vectors $\left(x_{m}\right)$ are initialized corresponding to the food source population, population size is set by scout bees, and other controlled parameters are also kept set. Let SS is the swarm size of the artificial bee colony (ABC) which randomly dispersed initial population of food sources. If $X_{i}$ is the solution among swarm and dimension is denoted by $M$, then

$$
X=\left[x_{i, 1}, x_{i, 2}, x_{i, 3}, \ldots, x_{i, M}\right] \text {. }
$$

Each and every employed bee will generate a new candidate solution in the neighbourhood of the current location. If new solution is represented by $Y_{i}$, where $X_{k}$ is the randomly chosen solution and random number is $\varphi_{i, j}$ which lies in between $[-1,1]$, then

$$
Y_{i, j}=x_{i, j}+\varphi_{i, j}\left(x_{i, j}-x_{k, j}\right) \text {. }
$$

A greedy selection is used once the new candidate solution $Y_{i}$ is generated. Now, the fitness value of $X_{i}$ is compared with $Y_{i}$. If $Y_{i}$ is better than $X_{i}$, then update $X_{i}$ with $Y_{i}$; otherwise, $X_{i}$ will be unchanged.

Once all employed bees will complete their search process, they share the food source details with onlooker bees via waggle dance. The onlooker bee tends to evaluate the nectar details received from all employed bees and selects that food source which has probability for its nectar amount. Roulette wheel selection mechanism is used for probabilistic choice. For swarm, the fitness value of the $i^{\text {th }}$ solution is $\mathrm{FIT}_{i}$ and is written as follows:

$$
P_{i}=\frac{F I T_{i}}{\sum F I T_{j}} .
$$

Food source will be assumed as abundant if position is not improved in defined number of cycles. Let us assume $X_{i}$ as an abundant source, then the new food source identified by the scout bee is replaced by the previous one, which is as follows:

$$
x_{i, j}=\mathrm{lb}_{j}+\operatorname{rand}(0,1)(\mathrm{ubj}-\mathrm{lbj}) .
$$

Here, $\mathrm{ub}$ and $\mathrm{lb}$ are the upper and lower boundaries of $j^{\text {th }}$ dimension

\section{Cuckoo Search (CS) Algorithm}

It is also a metaheuristic algorithm with good global search and fast convergence, proposed in the year 2009 [13]. In recent years, it has been applied to various applications where some process parameters need to be optimized. CS algorithm imitates the brood parasitism conduct of cuckoos and Levy flying actions of birds. It has extended search space because of a combination of recurrent short jumps and unplanned long jumps $[15,16]$. Some low-grade nests used to be abolished by probability discovery $\left(P_{a}\right)$, and new nests are created by a mechanism known as random walk. Fitness function $F_{i}$ needs to be calculated for every iteration by taking cuckoos randomly and accordingly best solution, i.e., nest with quality solution, is updated for ranking the solution.

\section{Vehicle Routing Problem (VRP)}

A general version of travelling salesman problem (TSP) is vehicle routing problem (VRP) [34, 35]. In this problem, vehicle tends to start and terminate service at the depot itself by providing service to all the customers. In VRP, multiple salesmen provide services to many consumers. Therefore, various parameters need to be optimized. Parameters for optimization may be time, distance, and cost of related routes [36-38]. Different variants of VRP are as follows:

(i) Capacitated vehicle routing problem (CVRP)

(ii) Time-dependent vehicle routing problem (TDVRP)

(iii) Vehicle routing problem with time windows (VRPTW)

(iv) Vehicle routing problem with heterogeneous fleet (VRPHF)

Vehicle routing problem with time windows (VRPTW) is a variation of capacitated vehicle routing problem where time duration is allocated to each consumer and the required services must be provided in that time window.

\section{Problem Description and Implementation}

A delivery center provides distribution on fresh products. Let us assume that there are $N$ service locations for food distributions and there are total $M$ delivery vehicles. Each 
and every delivery vehicle starts its service from the delivery center, and by covering all service locations, it reaches back to the delivery center. Each service location has specified time duration in which the delivery of fresh food must be done. Therefore, the objective is optimization for delivery vehicles of distributing fresh food products so that the required target can be achieved. Parameters for optimization for maintaining food quality can be shortest path travelled, finest service quality, and minimum cost of supply. This paper proposed a process for the timely distribution model of fresh food products route without any quality loss of food.

Quality loss function of perishable fresh products expresses the quality transformation with respect to time involved in the delivery process. Modelling is done by considering fresh products as perishable, and its temperature is kept constant during the entire delivery route. Metamorphic function is expressed as follows:

$$
X(t)=X_{0} \mathrm{Ke}^{-\beta t},
$$

where

\section{$t$ : delivery time}

$X_{0}$ : quality of fresh food product before delivery which is very good

$K$ : rate of food quality loss

$\beta$ : sensitivity of food products w.r.t. time (its value will be small when food products are highly sensitive otherwise vice versa)

If the customer is not satisfied with delivered food quality which is directly correlated with the time window problem, the penalty function needs to be introduced. Time window for penalty is being discussed in this paper. Let the time window is $\left[U_{i}, V_{i}\right]$ for satisfactory food delivery for each service location, and if food is delivered timely, i.e., within this window, the satisfaction level is met and no penalty is to be kept.

By considering various restrictions and for minimized cost of logistics distribution, the objective function for the fresh food distribution model can be written as follows:

$$
\min (Y)=X_{1}+X_{2}+X_{3}+X_{4},
$$

(i) $X_{1}$ : fixed cost

$$
\mathrm{X}_{1}=\mathrm{mF} \text {, }
$$

(ii) where $F$ is the fixed cost of each delivery vehicle which includes all kinds of expenses

(iii) $X_{2}$ : transportation cost

$$
X_{2}=\sum \sum \sum d_{i j} X_{i j K} C^{\prime},
$$

where $(i=0,1, \ldots, N),(j=0,1, \ldots, N)(K=0,1, \ldots, S)$

(iv) $X_{3}$ : goods lost cost

$$
X_{3}=\operatorname{pgiK}\left(1-e^{-\beta 0 t}\right) \text {. }
$$

(v) $X_{4}$ : punishment cost

$$
X_{4}=\theta_{1}\left(t_{i}-M_{i}\right)
$$

When each service location has one delivery vehicle for distribution of fresh food and all service locations use total $M$ delivery vehicles, it is mathematically written as

$$
\sum_{S \in M} B_{i S}=1 \quad \text { for } i \in N, \quad M \text { for } i=0 .
$$

Each delivery vehicle has only one route for food distribution, and summation of all distribution routes is small than the total number of delivery vehicles, i.e.,

$$
\sum_{S \in M} \sum_{j \in N} A_{i j S} \leq M, \quad \forall i=0 .
$$

Every delivery vehicle starts from a fresh logistic delivery center and ends its service there itself after accomplishing task; therefore, it is a closed delivery track.

$$
\sum_{j \in N} A_{j i s}=\sum_{j \in N} A_{j i s} \leq 1, \quad i=0, K=1,2,3, \ldots, M .
$$

The total stretch travelled by each delivery vehicle on each delivery route does not exceed the maximum stretch of vehicles.

$$
\sum_{i, j \in N} d_{i j} A_{i j K} \leq D, \quad \forall S \in M
$$

Total demand arriving at each delivery path must be less than the maximum capacity of each delivery vehicle.

$$
\sum_{j \in N} g_{i} B_{k i} \leq X_{L}, \quad S=1,2, \ldots, M
$$

\section{Results and Discussion}

In this paper, the artificial bee colony optimization algorithm is used for solving the discussed problem because this algorithm improves the convergence time. Various parameters for $\mathrm{ABC}$ algorithm are number of iterations, size of colony, limit value etc. The same problem is solved by applying one more swarm algorithm, i.e., cuckoo search algorithm. By varying the population size, results have been computed, and analysis has been done for optimality of result whether it depends on variable population size or increasing search space. Results have been tabulated for both algorithms, in two cases. The first case consists of 20 delivery locations, i.e., stores, and 5 delivery vehicles, and second case consists of 40 delivery locations and 10 delivery vehicles. Table 1 comprises the data corresponding to 20 delivery locations with 5 delivery vehicles for both $\mathrm{ABC}$ algorithm and CS algorithm. Table 2 comprises the data corresponding to 40 delivery locations with 10 delivery vehicles for both $\mathrm{ABC}$ algorithm and CS algorithm. Data related to each food delivery route, distance travelled, time required, and number of delivery locations, i.e., stores, have been enumerated in 
TABLE 1: 20 delivery locations with 5 delivery vehicles.

\begin{tabular}{lccccc}
\hline Algorithm & Route no. & Route travelled & Number of stores & Distance travelled (Km) & Travelling time (mins) \\
\hline & R1 & S-10-4-13-20-S & 4 & 6.04 & 51 \\
Artificial bee colony (ABC) & R2 & S-1-6-18-S & 3 & 4.51 & 42 \\
& R3 & S-3-11-8-12-14-19-S & 6 & 9.93 & 79 \\
& R4 & S-5-17-9-S & 3 & 4.48 & 44 \\
& R5 & S-15-16-7-2-S & 4 & 3.92 & 48 \\
\hline \multirow{5}{*}{ Cuckoo search (CS) } & R1 & S-1-18-19-S & 3 & 4.52 & 4.8 \\
& R2 & S-8-20-15-S & 3 & 10.09 & 49 \\
& R3 & S-17-10-14-7-12-3-S & 6 & 5.56 & 64 \\
& R4 & S-2-9-11-6-S & 4 & 5.49 & 59 \\
\hline
\end{tabular}

TABLE 2: 40 delivery locations with 10 delivery vehicles.

\begin{tabular}{|c|c|c|c|c|c|}
\hline Algorithm & Route no. & Route travelled & Number of stores & Distance travelled $(\mathrm{Km})$ & Travelling time (mins) \\
\hline \multirow{10}{*}{ Artificial bee colony (ABC) } & $\mathrm{R} 1$ & S-3-26-9-S & 3 & 5.23 & 42 \\
\hline & $\mathrm{R} 2$ & S-8-21-35-19-2-S & 5 & 6.52 & 61 \\
\hline & $\mathrm{R} 3$ & S-18-4-23-30-S & 4 & 5.89 & 46 \\
\hline & $\mathrm{R} 4$ & S-25-5-7-32-39-31-17-S & 7 & 11.3 & 93 \\
\hline & $\mathrm{R} 5$ & S-12-36-6-S & 3 & 5.44 & 38 \\
\hline & R6 & S-11-40-S & 2 & 4.34 & 31 \\
\hline & R7 & S-1-15-20-29-S & 4 & 5.91 & 51 \\
\hline & $\mathrm{R} 8$ & S-22-10-38-S & 3 & 5.29 & 42 \\
\hline & R9 & S-14-24-13-37-S & 4 & 5.88 & 48 \\
\hline & $\mathrm{R} 10$ & S-16-28-33-34-27-S & 5 & 6.6 & 58 \\
\hline \multirow{10}{*}{ Cuckoo search (CS) } & $\mathrm{R} 1$ & S-9-19-32-S & 3 & 4.98 & 41 \\
\hline & $\mathrm{R} 2$ & $S-1-10-7-26-S$ & 4 & 6.11 & 67 \\
\hline & $\mathrm{R} 3$ & S-28-21-14-8-2-S & 5 & 6.73 & 73 \\
\hline & $\mathrm{R} 4$ & S-11-5-18-23-29-30-38-S & 7 & 12.8 & 101 \\
\hline & $\mathrm{R} 5$ & S-17-36-40-S & 3 & 4.99 & 45 \\
\hline & R6 & S-31-13-25-S & 3 & 5.55 & 58 \\
\hline & R7 & S-16-22-37-S & 3 & 6.01 & 63 \\
\hline & $\mathrm{R} 8$ & S-12-39-33-S & 3 & 5.97 & 55 \\
\hline & $\mathrm{R} 9$ & $S-24-34-3-6-S$ & 4 & 5.88 & 60 \\
\hline & $\mathrm{R} 10$ & S-4-20-15-35-27-S & 5 & 6.82 & 84 \\
\hline
\end{tabular}

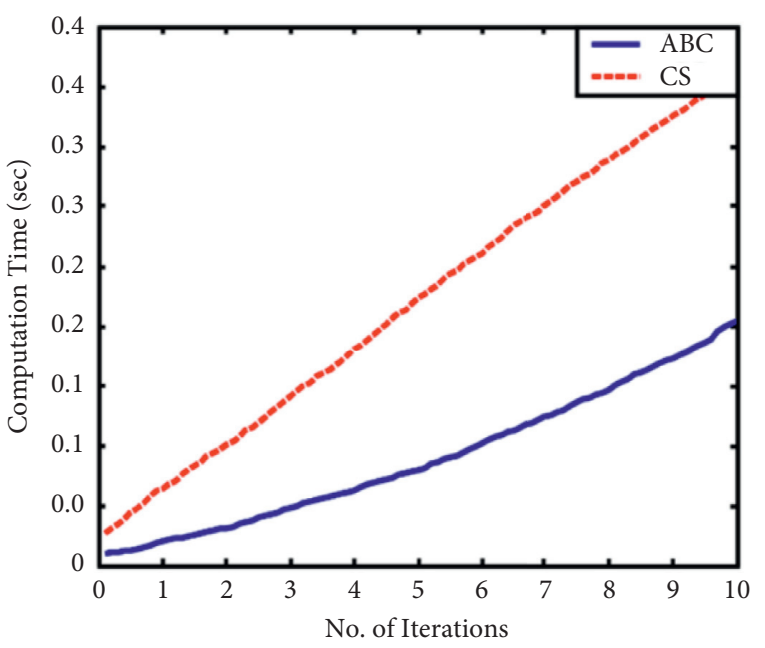

Figure 3: Convergence graph. 

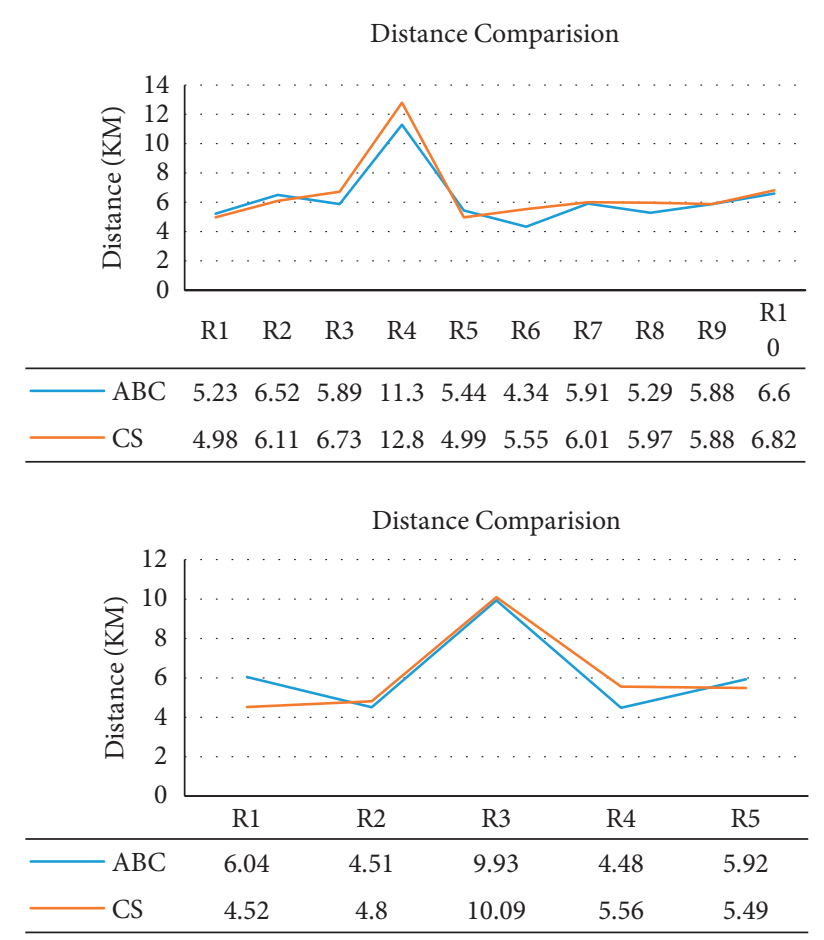
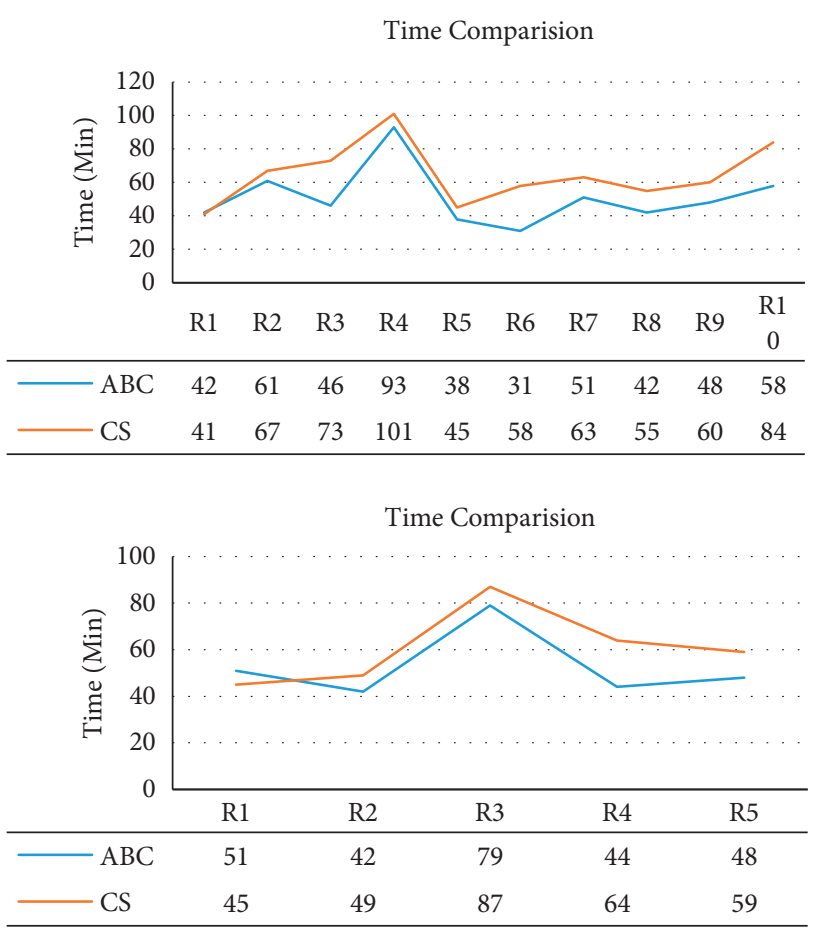

Figure 4: Comparison of distance travelled and time taken.

Tables 1 and 2. S denotes the initial location, i.e., depot from where vehicles will start and terminate their services.

Computation time is the time required by the algorithm to complete the task. Figure 3 shows the computation time for the artificial bee colony algorithm and cuckoo search algorithm for path planning of distribution of fresh food among various delivery locations without food quality loss and limited number of delivery vehicles. From Figure 3, it is clear that time required by the $\mathrm{ABC}$ algorithm is less than the $\mathrm{CS}$ algorithm; therefore, the performance of $\mathrm{ABC}$ algorithm is very much efficient and effective.

Figure 4 shows the distance travelled and time required for each route in both the cases, i.e., 40 delivery locations with 10 delivery vehicles and 20 delivery locations with 5 delivery vehicles using the artificial bee colony algorithm and cuckoo search algorithm. It can be observed that performance of the artificial bee colony algorithm is better in comparison with cuckoo search algorithm.

\section{Conclusion and Future Scope}

Food distribution within time window is always a research area for researchers in food industry because of its dynamic nature with lot many constraints. Reputation of the food industry and related owner depends upon the feedback of customers, which is directly associated with the quality of food and service. In this paper, fresh food delivery route optimization is applied on multiple delivery vehicles with multiple delivery locations including penalty function and quality loss function. $\mathrm{ABC}$ algorithm and CS algorithm are applied by varying the selection of function indicating the shortest route; therefore, food distribution is done within the time window. By analyzing all constraints, the overall process is examined, and path optimization is done and it has been observed that performance is better by using $A B C$ algorithm. Some of the limitations are still here because of not considering the dynamics of delivery vehicle and realtime traffic and other environment scenarios; therefore, further revision in the food distribution model can be done. Researchers suggest that further some more descriptive computational intelligence algorithms can be used to solve the same nature of problems, such as Monarch butterfly optimization (MBO), lion optimization algorithm (LOA), grasshopper optimization algorithm, earthworm optimization algorithm (EWA), elephant herding optimization (EHO), moth search (MS) algorithm, slime mould algorithm (SMA), Harris Hawks optimization (HHO), and butterfly optimization (BO). Some hybrid algorithms can also be applied to reduce other fresh food distribution limitations or reducing quality loss, and results can be compared to ascertain which algorithm works better. [28]

\section{Data Availability}

No data were used to support this study.

\section{Conflicts of Interest}

The authors declare that they have no conflicts of interest.

\section{References}

[1] Y. Liu, B. Guo, and C. Chen, "FooDNet: toward an optimized food delivery network based on spatial crowdsourcing," IEEE Transactions on Mobile Computing, vol. 18, 2019.

[2] M. Bortolini, M. Faccio, E. Ferrari, M. Gamberi, and F. Pilati, "Fresh food sustainable distribution: cost, delivery time and 
carbon footprint three-objective optimization," Journal of Food Engineering, vol. 174, pp. 56-67, 2016.

[3] Y. Zhang and X. D. Chen, "An optimization model for the vehicle routing problem in multi-product frozen food delivery," Journal of Applied Research and Technology, vol. 12, no. 2, pp. 239-250, 2014.

[4] W. Hu, H. Liang, C. Peng, B. Du, and Q. Hu, "A hybrid chaosparticle swarm optimization algorithm for the vehicle routing problem with time window," Entropy, vol. 15, no. 4, pp. 1247-1270, 2013.

[5] S. Wang, Z. Lu, and L. Wei, "Fitness-scaling adaptive genetic algorithm with local search for solving the Multiple Depot Vehicle Routing Problem," Simulation, vol. 92, pp. 601-616, 2016.

[6] H. L. Wang, "Distribution of perishable food based on models with time windows," Industrial Engineering Journal, vol. 11, no. 3, pp. 127-130, 2008.

[7] J. Brito, F. J. Martinez, J. A. Moreno, and J. L. Verdegay, "Fuzzy optimization for distribution of frozen food with imprecise times," Fuzzy Optimization and Decision Making, vol. 11, no. 3, pp. 337-349, 2012.

[8] M. Narayanasamy, J. Luke, and A. Rajan, "Comprehensive review on evolutionary optimization techniques applied for unit commitment problem," IEEE access, vol. 8, pp. 12980133014, 2020.

[9] Y. Aslam and N. Santhi, "A comprehensive survey on optimization techniques in image processing," Materials Today: Proceedings, vol. 24, no. 3, pp. 1758-1765, 2020.

[10] J. S. Arora, O. A. Elwakeil, A. I. Chahande, and C. C. Hsieh, "Global optimization methods for engineering applications: a review," Structural Optimization, vol. 9, no. 3-4, pp. 137-159, 1995.

[11] Y. M. Wang and H. L. Yin, "Cost-optimization problem with a soft time window based on an improved fuzzy genetic algorithm for fresh food distribution," Mathematical Problems in Engineering, vol. 201816 pages, 2018.

[12] M. Dorigo, M. Birattari, and T. Stutzle, "Ant colony optimization," IEEE Computational Intelligence Magazine, vol. 1, no. 4, pp. 28-39, 2006.

[13] A. H. Gandomi, X.-S. Yang, and A. H. Alavi, "Cuckoo search algorithm: a metaheuristic approach to solve structural optimization problems," Engineering with Computers, vol. 29, no. 1, pp. 17-35, 2013.

[14] A. li, "A particle swarm algorithm for inspection optimization in serial multi-stage processes," Applied Mathematical Modelling, vol. 36, no. 4, pp. 1455-1464, 2012.

[15] M. Mareli and B. Twala, "An adaptive Cuckoo search algorithm for optimisation," Applied Computing and Informatics, vol. 14, no. 2, pp. 107-115, 2018.

[16] A. S. Joshi, O. Kulkarni, G. M. Kakandikar, and V. M. Nandedkar, "Cuckoo search optimization-a review," Materials Today: Proceedings, vol. 4, no. 8, pp. 7262-7269, 2017.

[17] M. Yazdani and F. Jolai, "Lion optimization algorithm (LOA): a nature-inspired metaheuristic algorithm," Journal of Computational Design and Engineering, vol. 3, no. 1, pp. 24-36, 2016.

[18] S. Saremi, S. Mirjalili, and A. Lewis, "Grasshopper optimisation algorithm: theory and application," Advances in Engineering Software, vol. 105, pp. 30-47, 2017.

[19] C. Charin, D. Ishak, M. A. A. Mohd Zainuri, and B. Ismail, "Modified levy Flight optimization for a maximum power point tracking algorithm under partial shading," Applied Sciences, vol. 11, no. 3, 2021.
[20] A. Cheraghalipour, M. Hajiaghaei-Keshteli, and M. M. Paydar, "Tree Growth Algorithm (TGA): a novel approach for solving optimization problems," Engineering Applications of Artificial Intelligence, vol. 72, pp. 393-414, 2018.

[21] S. Mirjalili, S. M. Mirjalili, and A. Lewis, "Grey wolf optimizer," Advances in Engineering Software, vol. 69, pp. 46-61, 2014.

[22] T.-K. Dao, J.-S. Pan, T.-S. Pan, and T.-T. Nguyen, "Optimal path planning for motion robots based on bees pollen optimization algorithm," Journal of Information and Telecommunication, vol. 1, no. 4, pp. 351-366, 2017.

[23] A. A. Heidari, S. Mirjalili, H. Faris, I. Aljarah, M. Mafarja, and H. Chen, "Harris hawks optimization: algorithm and applications," Future Generation Computer Systems, vol. 97, pp. 849-872, 2019.

[24] A. Hammoudi, H. Ferhat, R. Y. Ali, G. Liang, and M. S. Sadiq, "A comparative analysis of the queuing search algorithm, the sine-cosine algorithm, the ant lion algorithm to determine the optimal weight design problem of a spur gear drive system," Materials Testing, vol. 63, no. 5, pp. 442-447, 2021.

[25] G. Dildar, P. Nantiwat, B. Sujin, M. S. Sadiq, and R. Y. Ali, “A novel hybrid water wave optimization algorithm for solving complex constrained engineering problems," Materials Testing, vol. 63, no. 6, pp. 560-564, 2021.

[26] A. Hammoudi, H. A. Ferhat, Y. Riza, and M. S. Sadiq, "Comparative investigation of the moth-flame algorithm and whale optimization algorithm for optimal spur gear design," Material Testing, vol. 63, no. 3, pp. 266-271, 2021.

[27] G. Wang, "Moth search algorithm: a bio-inspired metaheuristic algorithm for global optimization problems," Memetic Computing, vol. 10, no. 2, 2018.

[28] S. Li, H. Chen, M. Wang, A. A. Heidari, and S. Mirjalili, "Slime mould algorithm: a new method for stochastic optimization," Future Generation Computer Systems, vol. 111, pp. 300-323, 2020.

[29] A. H. Gandomi and A. H. Alavi, "Krill herd: a new bio-inspired optimization algorithm," Communications in Nonlinear Science and Numerical Simulation, vol. 17, no. 12, pp. 4831-4845, 2012.

[30] M. Tubishat, M. Alswaitti, S. Mirjalili, M. A. Al-Garadi, M. e. T. Alrashdan, and T. A. Rana, "Dynamic butterfly optimization algorithm for feature selection," IEEE Access, vol. 8, pp. 194303-194314, 2020.

[31] L. Zhang, S. Wang, K. Zhang et al., "Cooperative artificial bee colony algorithm with multiple populations for interval multiobjective optimization problems," IEEE Transactions on Fuzzy Systems, vol. 27, no. 5, pp. 1052-1065, 2019.

[32] D. Karaboga and B. Basturk, "On the performance of artificial bee colony (ABC) algorithm," Applied Soft Computing, vol. 8, no. 1, pp. 687-697, 2008.

[33] M. Koudil, K. Benatchba, A. Tarabet, and E. B. Sahraoui, "Using artificial bees to solve partitioning and scheduling problems in codesign," Applied Mathematics and Computation, vol. 186, no. 2, pp. 1710-1722, 2007.

[34] S. Katiyar, "Towards hybridization of nature inspired metaheuristic techniques for collision free motion planning," International Journal of Computer Science and Network, Technologies, vol. 7, no. 3, pp. 192-198, 2018.

[35] B. K. Patle, G. Babu L, A. Pandey, D. R. K. Parhi, and A. Jagadeesh, "A review: on path planning strategies for navigation of mobile robot," Defence Technology, vol. 15, no. 4, pp. 582-606, 2019. 
[36] W. Sun, Y. F. Lu, H. W. Tang, and M. Xue, "Path rules for mobile robots based on an improved A * algorithm," Journal of Hunan University, vol. 44, no. 2, pp. 94-101, 2017.

[37] K. Jeddisaravi, R. J. Alitappeh, L. C. Pimenta, and F. G. Guimarães, "Multi-objective approach for robot motion planning in search tasks," Applied Intelligence, vol. 45, no. 2, pp. 305-321, 2016.

[38] L. E. Kavraki, P. Svestka, J.-C. Latombe, and M. H. Overmars, "Probabilistic roadmaps for path planning in high-dimensional configuration spaces," IEEE Transactions on Robotics and Automation, vol. 12, no. 4, pp. 566-580, 1996. 